\title{
La santé de demain, déjà à la portée des médecins
}

Valérie Barbiéa ${ }^{\text {, Aitana Lebrand }}{ }^{a}$, Maïa Berman ${ }^{b}$

SIB Institut Suisse de Bioinformatique $-{ }^{a}$ Groupe de Bioinformatique Clinique; ${ }^{b}$ Département de Communication

Maladies héréditaires, infectieuses ou cancer: autant de patients qui bénéficient déjà des avancées en matière de santé personnalisée. Des défis existent néanmoins pour transformer les progrès technologiques en réalité médicale accessible à tous les praticiens. Eclairage et solutions au service de ces derniers en Suisse.

\section{Santé personnalisée: promesses et situation actuelle}

Les médecins de famille ont toujours adopté une approche personnalisée, du fait de leur connaissance du contexte de vie et des antécédents de santé de leur patient. Or de nouvelles techniques, disponibles depuis peu, leur permettront également, à terme, de puiser des informations dans le profil moléculaire de leur patient, en vue de la détection précoce de maladies ou de l'ajustement d'un traitement.

\section{Défis et solutions pour le praticien}

Les quantités massives de données génerées par les nouvelles technologies et la rapide évolution de ces dernières ont d'ores et déjà bouleversé l'écosystème clinique: de nouveaux métiers, tel que celui de bioinformaticien clinique, sont devenus essentiels au sein des laboratoires d'analyses, tandis que les hôpitaux font face à des besoins nouveaux en terme d'infrastructure informatique.

Une interdisciplinarité marquée en découle, et de fait une necessité de développer un langage commun entre divers experts - oncologues, pathologues, généticiens, bioinformaticiens - ainsi qu'un besoin en formation continue dédiée aux professionnels de la santé.

Pour répondre à ces défis, le SIB Institut Suisse de Bioinformatique (voir 1) travaille main dans la main avec les praticiens pour mieux comprendre et adresser leurs besoins, par exemple en collaborant avec les hôpitaux pour harmoniser les pratiques et la standardisation des données (voir 2); en développant des outils d'analyse de données pour l'amélioration du diagnostic en routine; ou encore en inaugurant un CAS en Oncologie Moléculaire Personnalisée avec les Hôpitaux universitaires de Bâle et de Lausanne (voir 3).

\footnotetext{
1. A propos du SIB

Organisation à but non lucratif fédérant 70 groupes de recherche et de services d'experts en bioinformatique en Suisse, l'institut fournit aux scientifiques et aux cliniciens une infrastructure, des ressources, une expertise et une formation de pointe en bioinformatique: www.sib.swiss.
}

2. Les données de patients, un casse-tête pour les chercheurs En amont des applications cliniques, la recherche est en pleine expansion. Avec, là aussi, des défis majeurs à relever, dont l'utilisation des données de patients. La Confédération a lancé, en 2017, une initiative nationale pour permettre à celles-ci d'être mises à disposition de projets de recherche - il s'agit du Swiss Personalized Health Network (SPHN). C'est au SIB qu'a été confiée la tâche d'établir les standards permettant l'échange de ces données, ainsi que de fournir une infrastructure informatique sécurisée aux projets de recherche.

3. Aller plus loin avec un Certificat d'Etudes Avancées (CAS) Premier du genre en Suisse, le CAS en Oncologie Moléculaire Personnalisée est ouvert à tout professionnel de la santé souhaitant acquérir ou approfondir ses connaissances sur les méthodologies utilisées pour générer, analyser et interpréter les profils moléculaires de patients, et sur les défis associés. La première session débutera en novembre 2018. Inscriptions et détails: www.pmo.unibas.ch 